\title{
Journal of \\ Geriatrics and Palliative Care
}

(C) All rights are reserved by Riquelme-Heras et al.

\section{Behavioral and Psychological Symptoms and Caregiver Burden in Mexican Alzheimer's Disease}

\section{Patients}

Keywords: Behavior problems; Alzheimer; Burden; Caregiver; Neuropsychiatric inventory

\begin{abstract}
Background: Alzheimer's disease accounts for $60-70 \%$ of dementias. An estimated 44 million people worldwide are living with dementia. Caregiver burden is a multidimensional response with physical, emotional, social and financial consequences for family members. Behavioral or neuropsychiatric problems are one of the most common causes that require medical attention and hospitalization. Their presence accentuates the deterioration of patients and increases the burden on caregivers.
\end{abstract}

Objective: To determine behavioral or neuropsychiatric problem that increase caregiver burden of family members with Alzheimer's disease.

Material and methods: Descriptive study, convenience sampling conducted in 60 primary caregivers of a family member with Alzheimer interviewed in Monterrey Alzheimer Association (Mexico), over 18 years old, in 2014

The Neuropsychiatric Inventory Questionnaire and the Zarit scale that values burden were applied. Zarit score and the different types of problems were obtained. The chi2 for categorical variables with a $p$ value of $<0.05$ was used.

Results: Caregivers were mostly women, of 53 years on average married, housewives, with college education. Caregiver burden in over half of the cases was moderate to severe; a third was intense and $15 \%$ mild to moderate. Statistically significant symptoms for caregiver burden were anxiety $(p<0.05)$, disinhibition $(p<0.05)$, irritability ( $p<$ 0.005 , hallucinations $(p<0.01)$

Conclusions: The patient behavior problems increase the caregiver burden.

\section{Background}

Dementia is a chronic disease with a decline in memory, thinking and behavior; progressive and irreversible, affecting the activities of daily living and quality of life. The International Alzheimer's Association estimates that 44 million people are living with dementia worldwide and these statistics will increase to 66 million by 2030 and 115 million by 2050 [1].

In Mexico more than 850,000 people ( $1 \%$ of the general population; 10.7 per 1,000 per year) are affected by the disease and approximately 2,030 patients die by it each year (4.5\% among those are over 60 years) [2]. These figures do not match reality. The actual numbers are unknown, so we can say that in our country the disease is under-diagnosed [3]. Alzheimer's disease (AD) accounts for 60 to $70 \%$ of dementias, and in the USA it is estimated that $13 \%$ of people over age 65 are affected [4]. Similar data were reported in other countries: in Spain the prevalence of dementia, AD and vascular

\section{Gómez-Gómez $\mathrm{C}^{1}$, Riquelme-Heras $\mathrm{H}^{1^{*}}$, Aranda- Galarza $I^{2}$, Gutiérrez-Herrera $\mathbf{R}^{3}$, Méndez-Espinosa $\mathrm{E}^{1}$ and Martínez-Lazcano $\mathrm{F}^{1}$}

${ }^{I}$ Family Medicine, University Hospital, Universidad Autónoma de Nuevo León, México

${ }^{2}$ Family Medicine, University Hospital, Universidad Autónoma de Nuevo León, México

${ }^{3}$ Family Medicine, University Hospital, Universidad Autónoma de Nuevo León, México

\section{*Address for Correspondence}

Riquelme-Heras H, Family Department, University Hospital, Universidad Autónoma de Nuevo León, Ave. Puerta del Sol 201, Depto. 401, Col. Dinastía, Monterrey, NL, Mexico, CP 64639; E-mail: riquelme@doctor.com

\section{Submission: 04 March, 2016}

Accepted: 15 June, 2016

Published: 20 June, 2016

Copyright: $\odot 2016$ Riquelme-Heras H, et al. This is an open access article distributed under the Creative Commons Attribution License, which permits unrestricted use, distribution, and reproduction in any medium, provided the original work is properly cited.

Reviewed \& Approved by: Dr. Craig S. Atwood, University of Wisconsin-Madison, USA

dementia amounted to 7.6, 4.6 and 1.8\% (Toledo Study) [5].

In Mexico there are few studies of Psychological and Behavioral Symptoms (PBS) and how these symptoms affect both patients and caregivers.

It is important that the primary care physician identify these symptoms early thus benefiting the patient and the caregiver.

The $\mathrm{AD}$ is considered as a major cause of dependence and it's called "the epidemic of the century", "it is among the first six conditions listed by the WHO as a priority in mental health" [6]. Factors that influence caregiver perceived burden include: caregiver characteristics such as kinship, gender, psychological resources and coping strategies; characteristics of people with dementia and cognitive status, behavior problems and characteristics of the context of care as duration, social support and family relationship's quality [7]. The concept of caregiver burden has multiple repercussions [8]. It includes physical, emotional, social and financial consequences for family members caring for a loved one with dementia [9].

Psychological and Behavioral Symptoms (PBS) in dementia have a high frequency in $\mathrm{AD}$, suggesting that they are part of the physiopathogeny of the dementia syndrome. Changes in functioning of cortical and subcortical areas (frontal and temporal) would be related to the most behavioral manifestations of the disease [10]. The PBS includes symptoms caused by disorders of perception, thought content, mood or behavior. The onset of these symptoms is manifested through significant changes in behavior and mood. Patients show changes in personality, irritability, anxiety or depression; hallucinations, delusions, aggression and wandering; they are developed in the early and middle stages of the disease [11]. 
Citation: Gómez GC, Riquelme-Heras H, Galarza Al, Herrera GR, Espinosa ME, et al. Behavioral and psychological Symptoms and Caregiver Burden in Mexican Alzheimer's Disease Patients. J Geriatrics Palliative Care 2016;4(1): 6.

The prevalence of psychotic symptoms in patients with dementia is about $66 \%$; the most frequent is persecutory delusions and one of its main features is that it can be triggered by episodes of verbal or physical aggression directed toward family and/or caregivers (Barcelona España) [12]. Anxiety is expressed by incessant questions regarding the events that will come, and a marked fear of being alone. Apathy includes loss of initiative and interest in daily activities and the reduction of emotional reactivity in social interaction. It is important to note that violent behaviors are those that produce more stress on caregivers and further compromise patient's quality of life [13].

The presence of PBS emphasizes the deterioration of patients while increasing the burden on caregivers, promoting premature institutionalization, so evaluation and treatment are essential to the welfare of patients and caregivers [10].

Neuropsychiatric manifestations of $\mathrm{AD}$ are common in clinical practice and are often associated in a syndromatic manner. For proper management a correct characterization of the behavior/problem and identification of the causal mechanism is essential. After discarding an underlying cause, it is advisable to treat antisocial behavior first. At the beginning it is suggested to test non-pharmacological measures and then, drugs with better efficacy and safety profile, selected according to the most relevant clinical symptom [14].

People with $\mathrm{AD}$ in the early stages may experience irritability, anxiety and depression; in final stages other symptoms such as sleep disturbances, verbal or physical outbursts, anxiety, nervousness, delusions and hallucinations may develop. Psychiatric and behavioral symptoms may be caused by a variety of factors called triggers, some ear infectious processes, urinary tract problems, among others, as well as side effects of some medications, and some circumstances that cause stress such as change of residence or caregiver. Identifying the causes helps to select the best intervention [15].

Both delusions and hallucinations become more frequent as the disease progresses. Several studies agree that the most common symptoms in advanced stages, such as agitation, aggression and wandering are also the most persistent over time. The psychopathological exam may be supplemented by the use of evaluation instruments [16].

The association between the frequency of behavioral problems and their impact on caregivers is high, especially in the social restrictions of caregivers. These caregiver restrictions condition an early institutionalization of the patient.

Perceptions of caregivers about behavioral problems are associated with the increased burden of care. Research in the area of behavioral problems and burden in $\mathrm{AD}$ is very important for the primary care physician. Caregiver burden in Mexico in AD is not well understood but it is estimated by studies that most caregivers suffer from burden related to care for a patient with Alzheimer's disease. Some of these studies report $80 \%$ mild to moderate burden [17] The PBS has an important role in this concept as well as pathologies associated with $\mathrm{AD}$. It is associated with the presence of diabetes, stroke, heart disease and depression [18].

In Latin-American culture, families have a moral obligation to care for loved ones, especially parents, grandparents and spouses. Most of the care is provided at home and women are generally responsible for it. Also, regardless of the number of family members, only one is the primary caregiver; besides the economic problems that the caregiver has to face.

There are several tools to assess the psychological and behavioral symptoms in patients with Alzheimer's disease; one of them is the Neuropsychiatric Inventory (Neuropsychiatric-Inventory-CaregiverDistress-Scale) (NPI-D) (Translated into Spanish) [19]. It is a useful tool for the family physician that provides a reliable assessment of behaviors commonly observed in patients with dementia. The questionnaire assesses the severity of the symptoms in the patient and the distress the caregiver experiences because of those symptoms. It rates the severity of the symptoms presented in the last month and how they affect the patient: $1=$ mild, symptoms are noticeable, but no significant changes, $2=$ moderate, the changes are significant, but not a dramatic change, and $3=$ severe, very marked or prominent; with dramatic changes. It also determines how the symptoms presented by the patient affect the caregiver: from $0=$ none to $5=$ severe or very severe (extremely distressing, unable to cope with). It rates 12 symptoms. The NPI-D is effective for assessing the progression and response to treatment.

The most frequently used instrument to measure caregiver burden is the Zarit Burden Interview (ZBI) [20]. This scale assesses subjectively the perceived burden of the caregiver and the answers are ranging from $0=$ "never" to $4=$ "always". The score ranges from $0=$ no burden to $61-88=$ severe burden.

The objective of the present research is to determine behavioral o neuropsychiatric problems that increase caregiver burden of a family member with Alzheimer's disease, and to identify the most common type of PBS.

\section{Material and Methods}

Design: Descriptive study, convenience sampling with structured face to face interviews of primary caregivers of a family member with $\mathrm{AD}$ that belong to a Monterrey Alzheimer Association in México; conducted in 2014. Sample size is determined for a confidence level of $95 \%$ and an accuracy of $5 \%$ resulting in a sample of 60 caregivers.

Caregivers were selected in each of the 6 Alzheimer Associations groups from a total of 464 caregivers. Surveys and instruments were applied by a family medicine resident, previously trained.

The instruments were applied at Alzheimer associations offices.

The application of the instruments was face to face.

Operational definition: Primary caregiver is the person who provides emotional and instrumental attention to a loved one, without compensation.

\section{Inclusion criteria}

- Over 18 years old.

- Primary caregiver of family member with AD.

- Agrees to participate. 
Citation: Gómez GC, Riquelme-Heras H, Galarza Al, Herrera GR, Espinosa ME, et al. Behavioral and psychological Symptoms and Caregiver Burden in Mexican Alzheimer's Disease Patients. J Geriatrics Palliative Care 2016;4(1): 6.

Table 1: Time of alzheimer's disease diagnosis

\begin{tabular}{|c|c|c|}
\hline Time of diagnosis & $\boldsymbol{f}$ & $\%$ \\
\hline Less than a year & 13 & 21.7 \\
\hline $\mathbf{1 - 5}$ years & $\mathbf{3 7}$ & $\mathbf{6 1 . 6}$ \\
\hline 6-9 years & 6 & 10.0 \\
\hline More than 10 years & 4 & 6.7 \\
\hline Total & $\mathbf{6 0}$ & $\mathbf{1 0 0 . 0}$ \\
\hline
\end{tabular}

Table 2: Caregiver time of care.

\begin{tabular}{|c|c|c|}
\hline Time of care & $\boldsymbol{f}$ & $\%$ \\
\hline Less than a year & 11 & 18.3 \\
\hline $\mathbf{1 - 5}$ years & $\mathbf{3 8}$ & $\mathbf{6 3 . 3}$ \\
\hline 6-9 years & 6 & 10.0 \\
\hline More than 10 years & 5 & 8.4 \\
\hline Total & $\mathbf{6 0}$ & $\mathbf{1 0 0 . 0}$ \\
\hline
\end{tabular}

Table 3: Alzheimer's patient disabilities.

\begin{tabular}{|c|c|c|}
\hline Disability & $\boldsymbol{f}$ & $\mathbf{\%}$ \\
\hline Motor & $\mathbf{1 6}$ & $\mathbf{2 6 . 7}$ \\
\hline Speech & 6 & 10.0 \\
\hline Vision & 4 & 6.7 \\
\hline Hearing & 3 & 5.0 \\
\hline Vision, hearing and movement & 2 & 3.3 \\
\hline Walk and speech & 1 & 1.7 \\
\hline Eating & 1 & 1.7 \\
\hline Vision and movement & 1 & 1.7 \\
\hline None & 26 & 43.2 \\
\hline Total & $\mathbf{6 0}$ & $\mathbf{1 0 0 . 0}$ \\
\hline
\end{tabular}

\section{Exclusion criteria}

- Primary caregivers of family members who have other types of dementia different than Alzheimer's.

- Primary caregivers who are not relatives to the person with Alzheimer's.

\section{Elimination criteria}

- Incomplete questionnaires.

Instruments: A questionnaire, divided into sections that include demographic data from both caregiver and person with dementia, disease characteristics and care were applied.

Behavior problems were measured by the Neuropsychiatric Inventory (Neuropsychiatric- Inventory- Caregiver- Distress- Scale) (NPI-D). The instrument has a scale between $0=$ none to $5=$ severe or very severe. It rates 12 symptoms.

The scale of caregiver burden ZBI was applied (Zarit, Orr and Zarit, 1985). The answers are ranging from $0=$ "never" to $4=$ "always". The sum of responses vary from $0=$ no burden to $61-88=$ severe burden, for each caregiver.

\section{Ethical issues}

The protocol was approved by Institutional Ethics and Research Committees, with MF14-005 code. Informed consent was obtained and confidentiality of information was guaranteed.

\section{Data analysis}

The data analysis was made in SPSS version 20 for Windows. Frequencies and percentages, means and standard deviation were obtained. Results of the Neuropsychiatric Inventory and Zarit scale were crossed. The $\mathrm{chi}^{2}$ for categorical variables with a $\mathrm{p}$ value of $<0.05$ as statistical significance was used.

\section{Results}

Caregiver socio-demographic characteristics: They are mostly married women, average age 53, college educated, housewives, Catholic, with medical insurance and with high income. They have less than 5 years of caring for, usually the mother, 7 days a week, 24 hours a day. A third part of them receive support in caring from 2 people, usually siblings. Half of them are healthy and those who are sick have hypertension and diabetes.

Table 4: Pathologies associated with caregiver burden in alzheimer's disease.

\begin{tabular}{|c|c|c|}
\hline Pathologies & $\boldsymbol{F}$ & \% \\
\hline Diabetes Mellitus & $\mathbf{1 2}$ & $\mathbf{2 0 . 0}$ \\
\hline Hypertension & 9 & 15.0 \\
\hline Cardiovascular & 5 & 8.3 \\
\hline Diabetes and hypertension & 4 & 6.7 \\
\hline Neurological & 3 & 5.0 \\
\hline Hypertension and urinary incontinence & 1 & 1.7 \\
\hline Other & 25 & 41.6 \\
\hline None & 1 & 1.7 \\
\hline
\end{tabular}

$n=60(p=0.01)$

Table 5: Main neuropsychological symptoms in alzheimer's patients.

\begin{tabular}{|c|c|c|}
\hline \multirow{2}{*}{ Symptoms } & \multicolumn{2}{|c|}{ Total } \\
\cline { 2 - 3 } Delusions & $f$ & $\%$ \\
\hline Hallucinations & 43 & 71.7 \\
\hline Agitation or aggression & 31 & 51.7 \\
\hline Depression or dysphoria & 28 & 46.7 \\
\hline Anxiety & 35 & 58.3 \\
\hline Elation or euphoria & 45 & 75.0 \\
\hline Apathy or indifference & 11 & 18.3 \\
\hline Disinhibition & 43 & 71.7 \\
\hline Irritability and lability & 40 & 66.7 \\
\hline Motor & 37 & 61.7 \\
\hline Night time & 31 & 51.7 \\
\hline Appetite and eating & 30 & 50.0 \\
\hline & 26 & 43.3 \\
\hline
\end{tabular}


Citation: Gómez GC, Riquelme-Heras H, Galarza Al, Herrera GR, Espinosa ME, et al. Behavioral and psychological Symptoms and Caregiver Burden in Mexican Alzheimer's Disease Patients. J Geriatrics Palliative Care 2016;4(1): 6.

ISSN: 2373-1133

Table 6: Neuropsychiatric inventory symptoms and burden.

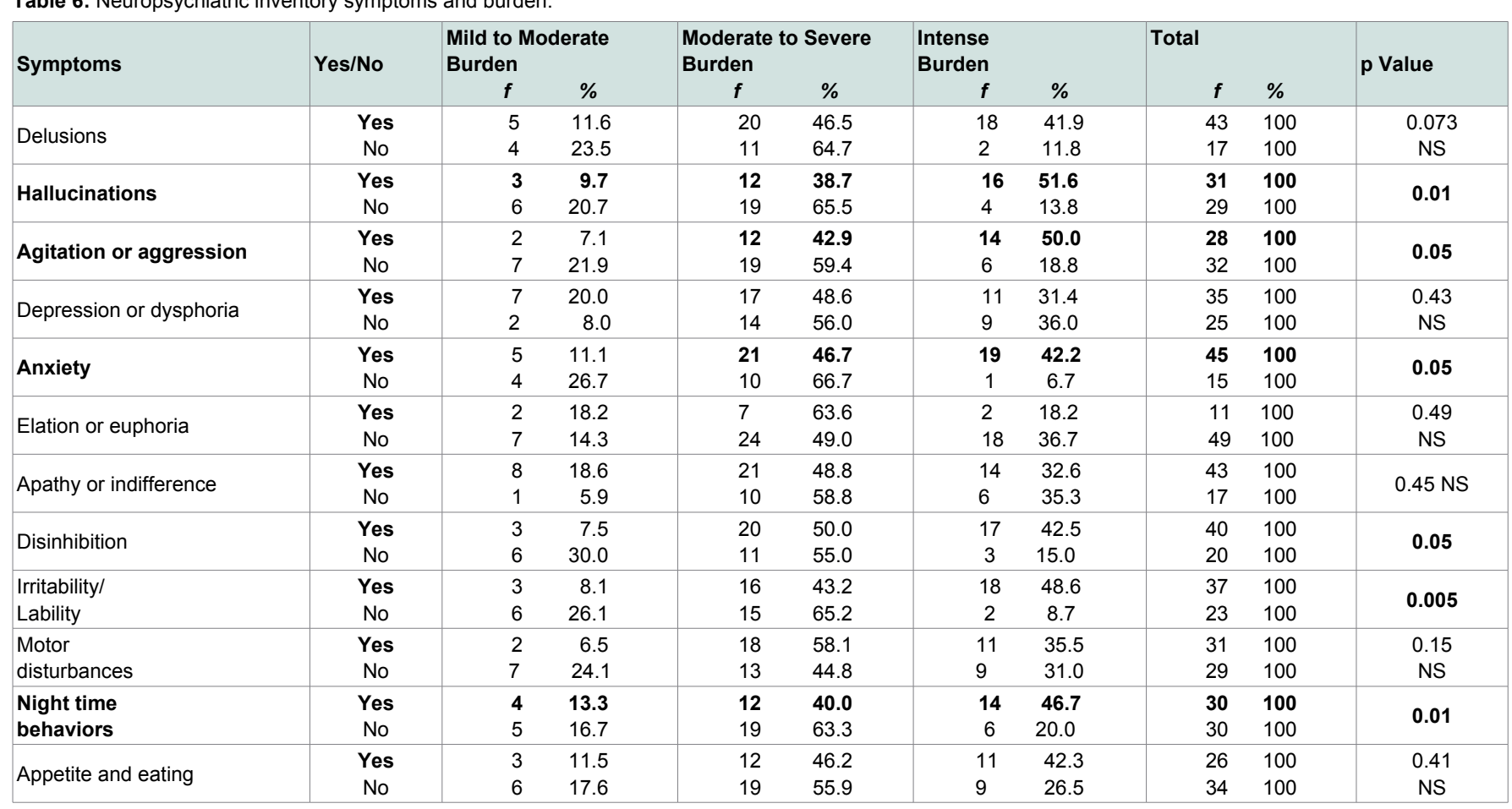

$\mathrm{n}=60$.

Elderly socio-demographic characteristics: They are mostly women, average age 78 , more than half are widows, elementary education, housewives, medical insurance, Catholic, a third part without income. For $58 \%$ of them live at home and $42 \%$ in the caregiver house. For $63.3 \%$ the mother of the caregiver is the one who has dementia, spouse $15 \%$ and in $13.3 \%$ is the father. Only $13 \%$ has been hospitalized once in the past year.

The average years since the $\mathrm{AD}$ diagnosis was established are 1-5 years; so is the time that the caregiver has devoted to his/her relative (1-5 years) (Tables 1 and 2).

The most common type of disability in patients with $\mathrm{AD}$ is motor disability (26.7\%) followed by speech, vision and hearing and/or some combinations (Table 3 ).

Pathologies found in this group of patients were: diabetes mellitus $20 \%$, hypertension $15 \%$, cardiovascular diseases $8.3 \%$, diabetes and hypertension $6.7 \%$, neurological disease $5 \%$, hypertension and urinary incontinence $1.7 \%$; other disease $41.6 \%$; none $1.7 \%$. Pathologies associated with $\mathrm{AD}$ are significantly correlated with caregiver burden $(\mathrm{p}=0.01)($ Table 4$)$

Main neuropsychological symptoms in Alzheimer's patients are shown in Table 5 (Anxiety 75.0\%, Delusions 71.7\%, Apathy or indifference $71.7 \%)$.

Neuropsychiatric Inventory results can be seen in Table 6, from which: delusions, hallucinations, agitation or aggression, depression or dysphoria, anxiety, apathy or indifference, stand out among others, and are related to caregiver burden. The symptoms associated with caregiver burden with a strong statistical correlation are: anxiety $\mathrm{p}<$ 0.05 ; hallucinations $\mathrm{p}<0.01$; nocturnal behavior, with $\mathrm{p}<0.01$; and agitation or aggression with $\mathrm{p}<0.05$.

All caregivers surveyed reported burden via the Zarit questionnaire, (100\%).

The most important degree of burden was moderate to severe, (51.7\%).

Finally, Table 7 shows the frequency of burden.

\section{Discussion}

In dimensions of care, caregivers often get help with instrumental activities of daily living especially from family members, demonstrating the importance of sharing care with other people and to avoid caregiver burden [21]. In the study, $26.7 \%$ of caregivers do not receive any kind of help; $28.3 \%$ only received help from one person and $33.3 \%$ from two; highlighting, as the international literature shows, that few people are in charge of caring for a loved one with dementia, which increases the burden. When a caregiver receives help, the sister provided it in $31.7 \%$ of cases and the brother in $8.3 \%$. Caregivers take care of his/her mother in $63.3 \%$, the father in $13.3 \%$ and $15 \%$ care for the spouse.

Table 7: Zarit scale results. Caregiver burden.

\begin{tabular}{|c|c|c|}
\hline Caregiver burden & $\boldsymbol{f}$ & $\%$ \\
\hline No burden & 0 & 0 \\
\hline Mild to moderate & 9 & 15.0 \\
\hline Moderate to severe & $\mathbf{3 1}$ & $\mathbf{5 1 . 7}$ \\
\hline Intense & 20 & 33.3 \\
\hline Total & $\mathbf{6 0}$ & $\mathbf{1 0 0 . 0}$ \\
\hline
\end{tabular}


Citation: Gómez GC, Riquelme-Heras H, Galarza Al, Herrera GR, Espinosa ME, et al. Behavioral and psychological Symptoms and Caregiver Burden in Mexican Alzheimer's Disease Patients. J Geriatrics Palliative Care 2016;4(1): 6.

ISSN: 2373-1133

In regard to psychological and behavioral problems in the Neuropsychiatric Inventory, anxiety, apathy or indifference, delusions, disinhibiting, irritability and depression prevailed among other symptoms commonly observed. In Spain, a study found the most common symptoms were apathy, depression, anxiety and emotional lability [12]. In patients with dementia, severity of symptoms was moderate, it means that the changes are significant but not dramatic and the caregiver distress was also moderate, so it's stressful and not always easy to handle.

In the present study, in $\mathrm{AD}$ patients, the presence of behavior problems as hallucinations was $48 \%$ and in a study conducted in Spain it was $17 \%$; agitation $48 \%$ to $31 \%$ and depression $58 \%$ to $37 \%$ [21]. In another European study in the Netherlands the most frequent PBS associated with dementia were agitation for $31 \%$, apathy, and irritability with $34 \%$ [22].

Dr. Cleusa Ferri mentions "Little is known about the prevalence of, or associations with behavioral and psychological symptoms of dementia (BPSD) in developing countries". The most common symptoms found in the countries studied were: Depression syndromes (43.8\%) followed by anxiety neurosis (14.2\%) and schizophreniform/ paranoid psychosis (10.9\%) [23].

The presentation of certain behavioral problems such as hallucinations, agitation or aggression, anxiety, disinhibiting, irritability or lability and nocturnal behavior problems are significantly related to increased caregiver burden. It is understandable, because they are more difficult to handle and require more people and time to care.

The Zarit scale score was 56.1 corresponding to a moderate to severe burden. The highest score corresponded to the fact that the relative with dementia depends on the caregiver, fear for the future that awaits the loved one and the stress involved in the care; in addition to other responsibilities. The burden of caring is widely documented in the literature.

In a study to measure the burden of caregivers in Mexico, $81 \%$ was moderate to severe against the $51 \%$ reported in the present study [17].

Pathologies associated with AD in our study were significant in increasing caregiver burden $(\mathrm{p}=0.01)$, as was reported in another study from Mexico [18].

No statistical significance was observed between burden and gender, time of care, hours a day of care or time of diagnosis of the disease as it should be expected, perhaps due to sample size or population characteristics.

Educating the general public about the causes of Alzheimer's and behavioral manifestations of the disease can reduce the burden. A better understanding of the causes and effects of burden can lead to interventions that reduce their impact.

Knowledge of PBS can help caregivers to seek support from family and friends and formal services, so they can lessen the burden.

Additional negative outcomes for caregivers, such as depression and physical illness can occur and cause premature placement of patients with dementia in nursing homes; and all of this leads to higher costs of health care.

\section{Conclusions}

The PBS which increase caregiver burden are anxiety, disinhibiting, irritability, hallucinations and nocturnal behavior.

All caregivers surveyed reported some burden degree. The most important degree of burden was moderate to severe.

Pathologies associated with $\mathrm{AD}$ as diabetes, hypertension and cardiovascular disease, were significant in increasing caregiver burden.

The effectiveness of clinical interventions based on evidence can increase significantly if the behavioral problems and caregiver burden are properly evaluated.

\section{References}

1. Bilbul M, Schipper HM (2011) Risk profiles of alzheimer disease. Can $J$ Neurol Sci 38: 580-592.

2. Gutiérrez LM, Ostrosky F, Sánchez S, Villa A (2001) Prevalence of dementia and mild cognitive impairment in subjects 65 years older in Mexico City: An Epidemiological Survey. Gerontology 47: 145.

3. Mejía-Arango S, Miguel-Jaimes A, Villa A, Ruiz-Arregui L, Gutiérrez-Robledo LM (2007) Deterioro cognoscitivo y factores asociados en adultos mayores en México. Salud Pública México 49: 475-481.

4. Uzun S, Kozumplik O, Folnegović-Šmalc V (2011) Alzheimer's dementia: current data review. Coll Antropol 35: 1333-1337.

5. García García FJ, Sánchez Ayala MI, Pérez Martín A, Martín Correa E Marsal Alonso C, et al. (2001) The prevalence of dementia and its main subtypes in subjects older than 65 years: impact of occupation and education. The Toledo Study. Med Clin (Barc) 116: 401-407.

6. Wortmann M (2012) Dementia: a global health priority - highlights from an ADI and World Health Organization report. Alzheimers Res Ther 4: 40.

7. Etters L, Goodall D, Harrison BE (2008) Caregiver burden among dementia patient caregivers: a review of the literature. J Am Acad Nurse Pract 20: 423 428.

8. Carretero S, Garcés J, Ródenas F, Sanjosé V (2009) The informal caregiver's burden of dependent people: theory and empirical review. Arch Geronto Geriatr 49: 74-79.

9. Zarit SH, Reever KE, Bach-Peterson J (1980) Relatives of the impaired elderly: correlates of feelings of burden. Gerontologist 20: 649-655.

10. García-Alberca JM, Lara Muñoz JP, Berthier Torres M (2010) Neuropsychiatric and behavioral symptomatology in Alzheimer disease. Actas Esp Psiquiatr 38: 212-222.

11. Chang KH, Horrocks S (2006) Lived experiences of family caregivers of mentally ill relatives. J Adv Nurs 53: 435-443.

12. López-Pousa S, Vilalta-Franch J, Garre-Olmo J, Pons S, Cucurella MG (2007) Caracterización y prevalencia de los síntomas psicológicos y conductuales en pacientes con demencia. Rev Neurol 45: 683-688.

13. Matusevich D, Ruiz M, Finkelstein C, Vairo C (2002) Síntomas conductuales y psicológicos de los pacientes con demencia. Evid Aten Primaria 5: 177-179.

14. Slachevsky Ch A, Fuentes GP (2005) Síntomas psicológicos y conductuales asociados a demencias: actualización terapéutica. Rev Méd Chile 133: 1242 1251.

15. García-Alberca JM, Lara JP, González-Barón S, Barbancho MA, Porta D, et al. (2008) Prevalencia y comorbilidad de síntomas neuropsiquiátricos en la enfermedad de alzheimer. Actas Esp Psiquiatr 36: 265-270.

16. Castiñeiras AR, Díaz Grávalos GJ, Hermida SR, González AL, Gil CV et al. (2012) Prevalencia de síntomas neuropsiquiátricos en pacientes institucionalizados con diagnóstico de demencia y factores asociados: estudio transversal multicéntrico. Rev Esp Geriatría Gerontol 47: 96-101. 
Citation: Gómez GC, Riquelme-Heras H, Galarza Al, Herrera GR, Espinosa ME, et al. Behavioral and psychological Symptoms and Caregiver Burden in Mexican Alzheimer's Disease Patients. J Geriatrics Palliative Care 2016;4(1): 6.

ISSN: 2373-1133

17. Domínguez-Sosa G, Zavala-Gonalez MA,Cruz-Mendez DC, RamírezRamírez MO (2010) Síndrome de sobrecarga en cuidadores primarios de adultos mayores de Cárdenas, Tabasco, México. Enero a mayo de 2008. Revista Médicas UIS 23: 28-37.

18. Mejía-Arango S, Miguel-Jaimes A, Villa A, Ruiz-Arregui L, M en C, et al (2007) Deterioro cognoscitivo y factores asociados en adultos mayores en México. Salud Pública de México 49: S475-S481.

19. Kaufer DI, Cummings JL, Christine D, Bray T, Castellón S, et al. (1998) Assessing the impact of neuropsychiatric symptoms in alzheimer's disease: the neuropsychiatric inventory caregiver distress scale. J Am Geriatr Soc 46 210-215.
20. Zarit SH, Orr NK, Zarit JM (1985) Families under stress: caring for the patient with alzheimer's disease and related disorders. University Press, New York.

21. Robinson KM, Adkisson P, Weinrich S (2001) Problem behavior, caregiver reactions, and impact among caregivers of persons with alzheimer's disease. J AdvNurs 36: 573-582.

22. Zuidema SU, Derksen E, Verhey FR, Koopmans RT (2007) Prevalence of neuropsychiatric symptoms in a large sample of Dutch nursing home patients with dementia. Int J Geriatr Psychiatry 22: 632-638.

23. Ferri CP, Ames D, Prince M; 10/66 Dementia Research Group (2004) Behavioral and psychological symptoms of dementia in developing countries. Int Psychogeriatr 16: 441-459. 